This item was submitted to Loughborough's Research Repository by the author.

Items in Figshare are protected by copyright, with all rights reserved, unless otherwise indicated.

\title{
Theory of giant and nil proximity effects in cuprate semiconductors
}

PLEASE CITE THE PUBLISHED VERSION

PUBLISHER

(C) American Physical Society

VERSION

VoR (Version of Record)

LICENCE

CC BY-NC-ND 4.0

REPOSITORY RECORD

Alexandrov, A.S.. 2019. "Theory of Giant and Nil Proximity Effects in Cuprate Semiconductors". figshare. https://hdl.handle.net/2134/4276. 
This item was submitted to Loughborough's Institutional Repository (https://dspace.lboro.ac.uk/) by the author and is made available under the following Creative Commons Licence conditions.

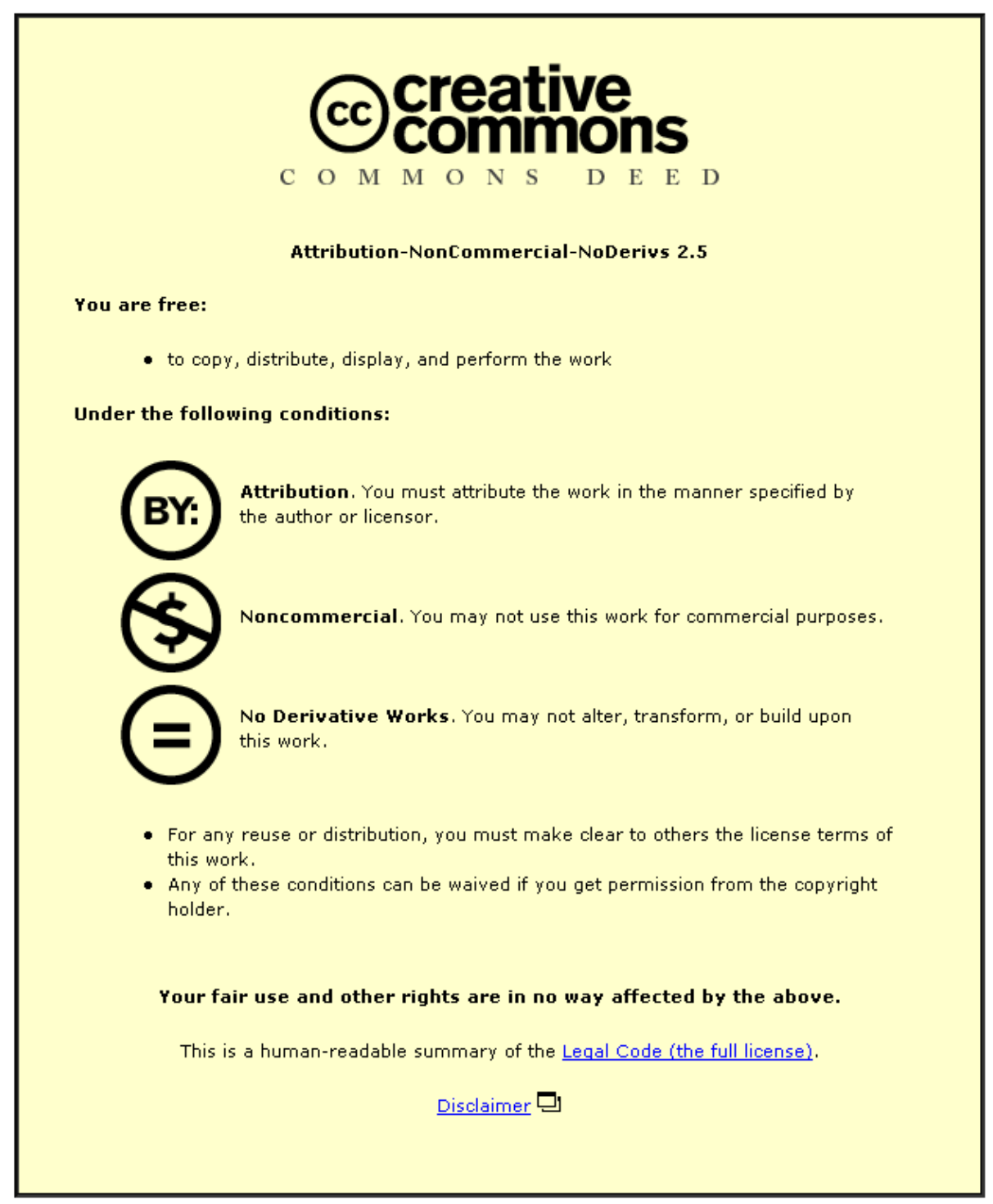

For the full text of this licence, please go to: http://creativecommons.org/licenses/by-nc-nd/2.5/ 


\title{
Theory of giant and nil proximity effects in cuprate semiconductors
}

\author{
A. S. Alexandrov \\ Department of Physics, Loughborough University, Loughborough LE11 3TU, United Kingdom
}

(Received 15 January 2007; published 9 April 2007)

\begin{abstract}
A number of observations point to the possibility that high- $T_{c}$ cuprate superconductors may not be conventional Bardeen-Cooper-Schrieffer superconductors but rather derive from the Bose-Einstein condensation of real-space pairs, which are mobile small bipolarons. A solution of the Gross-Pitaevskii equation describing Bose-condensate tunneling into a cuprate semiconductor is analytically found. It accounts qualitatively and quantitatively for nil and giant proximity effects discovered experimentally in cuprates.
\end{abstract}

DOI: 10.1103/PhysRevB.75.132501 PACS number(s): 71.38. $-\mathrm{k}$, 72.15.Jf, 74.40.+k, 74.72. $-\mathrm{h}$

Perhaps the most striking property of cuprate superconductors is the giant proximity effect (GPE). Several groups ${ }^{1}$ reported that in the Josephson cuprate $S N S$ junctions, supercurrent can run through normal $N$ barriers, with the thickness $2 L$ greatly exceeding the coherence length, when the barrier is made from a slightly doped nonsuperconducting cuprate (the so-called $N^{\prime}$ barrier). Using an advanced molecularbeam epitaxy, Bozovic et al. ${ }^{1}$ proved that GPE is intrinsic, rather than caused by any extrinsic inhomogeneity of the barrier. Resonant scattering of soft-X-ray radiation did not find any signs of intrinsic inhomogeneity (such as charge stripes, charge-density waves, etc.) either. ${ }^{2}$ Hence, GPE defies the conventional explanation, which predicts that the critical current should exponentially decay with the characteristic length of about the coherence length, $\xi \lesssim 1 \mathrm{~nm}$ in cuprates. $^{3}$ Annealing the junctions at low temperatures in vacuum rendered the barrier insulating. Remarkably, when the $S N^{\prime} S$ junction was converted into a superconductorinsulator-superconductor device, no supercurrent was observed, even in devices with the thinnest (one unit cell thick) barriers ${ }^{4}$ [nil proximity effect (NPE)].

Cuprate superconductors with low density of free carriers and poor mobility (at least in one direction) are characterized by poor screening of high-frequency $c$-axis polarized optical phonons. The unscreened Fröhlich interaction between oxygen holes and these phonons combined with on-site repulsive correlations (Hubbard $U$ ) binds holes into superlight intersite bipolarons, ${ }^{5}$ which are real-space pairs dressed by phonons. Experimental evidence for exceptionally strong electronphonon interactions is now so overwhelming ${ }^{6-10}$ that a bipolaronic charged Bose gas ${ }^{11}$ (CBG) could be a feasible alternative to the Bardeen-Cooper-Schrieffer (BCS)-like scenarios of cuprates. The bipolaron theory predicted some key features of cuprate superconductors, such as anomalous upper critical fields, spin and charge pseudogaps, and unusual isotope effects later discovered experimentally. The theory explained normal-state kinetics, including the anomalous Hall-Lorenz number, high $T_{c}$ values, specific-heat anomalies of cuprates (for a review, see Ref. 11), and more recently the $d$-wave symmetry of the order parameter in underdoped ${ }^{12}$ and overdoped ${ }^{13}$ samples, the normal-state Nernst effect, ${ }^{14}$ and diamagnetism. ${ }^{15}$

Here, I show that both GPE and NPE can be broadly understood as the Bose-Einstein condensation (BEC) tunneling into a cuprate semiconductor.

A stationary condensate wave function of $\mathrm{CBG}, \psi(\mathbf{r})$, obeys the following equation:

$$
\left[-\frac{\Delta}{2 m}-\mu+V(\mathbf{r})\right] \psi(\mathbf{r})=0,
$$

where $m$ is the boson mass, $\mu$ is the chemical potential, and $V(\mathbf{r})$ describes the Coulomb and hard-core composed bosonboson repulsions and their attraction to a neutralizing charge background (here and further $\hbar=c=k_{B}=1$ ). While the electric-field potential can be found from the corresponding Poisson-like equation, ${ }^{16}$ a solution of two coupled nonlinear differential equations for the order parameter $\psi(\mathbf{r})$ and for the potential $V(\mathbf{r})$ is a nontrivial mathematical problem, which requires a "multishooting" numerical approach. Here, we restrict our analysis by a short-range potential $V(\mathbf{r})$ $=V|\psi(\mathbf{r})|^{2}$, where a constant $V$ accounts for the hard-core and screened Coulomb repulsions. Then, Eq. (1) is the familiar Gross-Pitevskii (GP) equation. ${ }^{17}$ In the tunneling geometry of $S N^{\prime} S$ junctions (Figs. 1 and 2), it takes the form

$$
\frac{1}{2 m_{c}} \frac{d^{2} \psi(Z)}{d Z^{2}}=\left[V|\psi(Z)|^{2}-\mu\right] \psi(Z),
$$

in the superconducting region, $Z<0$, near the $S N$ boundary (Fig. 1). Here, $m_{c}$ is the boson mass in the direction of tunneling along $Z$. Deep inside the superconductor, $|\psi(Z)|^{2}=n_{s}$ and $\mu=V n_{s}$, where the condensate density $n_{s}$ is about $x / 2$, if the temperature is well below $T_{c}$ of the superconducting electrode. Here, the in-plane lattice constant $a$ and the unit cell volume are taken as unity, and $x$ is the doping level as in $\mathrm{La}_{2-x} \mathrm{Sr}_{x} \mathrm{CuO}_{4}$.

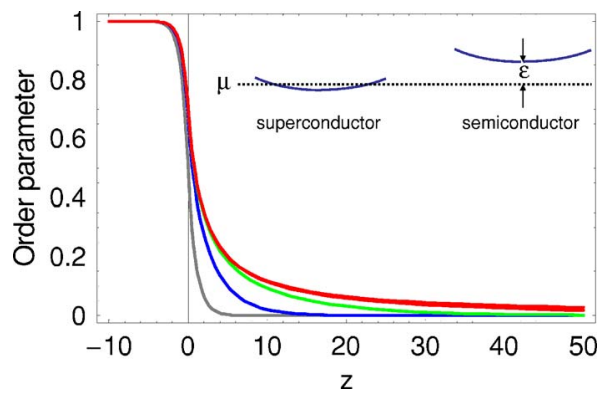

FIG. 1. (Color online) BEC order parameter at the $S N$ boundary for $\tilde{\mu}=1.0,0.1,0.01$, and $\leqslant 0.001$ (upper curve). The chemical potential is found above the boson band edge due to the boson-boson repulsion in cuprate superconductors and below the edge in cuprate semiconductors with low doping. 


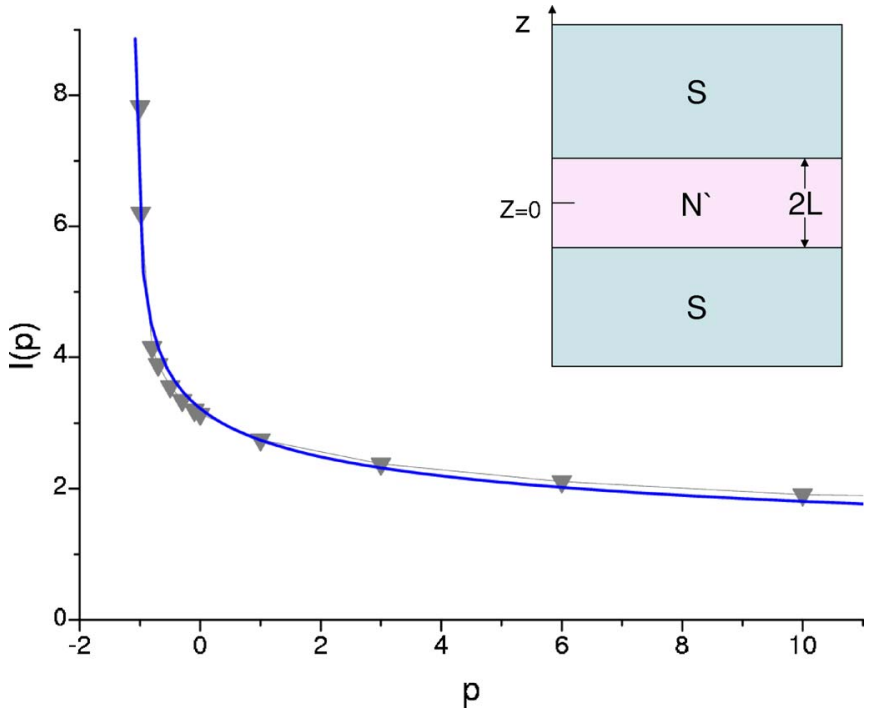

FIG. 2. (Color online) Numerical values of the integral $I(p)$ in Eq. (18) (symbols) compared with the analytical approximation (solid line). Inset shows schematically the trilayer $S N^{\prime} S$ device of Ref. 1.

The normal barrier at $Z>0$ is an underdoped cuprate above its transition temperature, $T_{c}^{\prime}<T$, where the chemical potential $\mu$ lies below the bosonic band by some energy $\epsilon$ (Fig. 1) found from $\int d E N(E)[\exp ((E+\epsilon) / T)-1]^{-1}=x^{\prime} / 2$. Here, $N(E)$ is the bipolaron density of states (DOS), and $x^{\prime}<x$ is the doping level of the barrier. In-plane bipolarons are quasi-two dimensional repulsive bosons, propagating along the $\mathrm{CuO}_{2}$ planes with the effective mass $m$ several orders of magnitude smaller than their out-of-plane mass, $m_{c} \gg m .^{11}$ Using bipolaron band dispersion, $E(\mathbf{K})$ $=K^{2} / 2 m+2 t_{c}\left[1-\cos \left(K_{\perp} d\right)\right]$, the DOS is found as $N(E)$ $=\left(m / 2 \pi^{2}\right) \arccos \left(1-E / 2 t_{c}\right)$ for $0<E<4 t_{c}$, and $N(E)$ $=m / 2 \pi$ for $4 t_{c}<E$. Here $K$ and $K_{\perp}$ are the in-plane and out-of-plane center-of-mass momenta, respectively, $t_{c}$ $=1 / 2 m_{c} d^{2}$, and $d$ is the interplane distance. As a result, one obtains

$$
\epsilon(T) \leqslant-T \ln \left(1-e^{-T_{0} / T}\right)
$$

which is exponentially small at $T_{c}^{\prime}<T \ll T_{0}$ turning into zero at $T=T_{c}^{\prime}$, where $T_{c}^{\prime} \approx T_{0} / \ln \left(T_{0} / 2 t_{c}\right)$ and $T_{0}=\pi x^{\prime} / m \gg T_{c}^{\prime} \gg t_{c}$.

It is important to note that $\epsilon(T)$ remains also small at $T_{c}^{\prime}(2 \mathrm{D}) \leqslant T \ll T_{0}$ in the purely two-dimensional (2D) repulsive Bose gas. ${ }^{18}$ While in two dimensions Bose condensation does not occur in either the ideal or the interacting system, there is a phase transition to a superfluid state at $T_{c}^{\prime}(2 \mathrm{D})$ $=T_{0} / \ln \left(1 / f_{0}\right) \ll T_{0}$, where $f_{0} \ll 1$ depends on the density of hard-core dilute bosons and their repulsion. ${ }^{18,19}$ The superfluid transition takes place only if there is a residual repulsion between bosons, i.e., $T_{c}^{\prime}(2 \mathrm{D})=0$ for the ideal 2D Bose gas. Actually, $T_{c}^{\prime}(2 \mathrm{D})$ gives a very good estimate for the exact Berezinski-Kosterlitz-Thouless (BKT) critical temperature in the dilute Bose gas, where the BKT contribution of vertices is important only very close to $T_{c}^{\prime}(2 \mathrm{D}) .{ }^{19}$

Then, the GP equation in the barrier is written as

$$
\frac{1}{2 m_{c}} \frac{d^{2} \psi(Z)}{d Z^{2}}=\left[V|\psi(Z)|^{2}+\epsilon\right] \psi(Z) .
$$

Introducing the bulk coherence length $\xi=1 /\left(2 m_{c} n_{s} V\right)^{1 / 2}$ and dimensionless $f(z)=\psi(Z) / n_{s}^{1 / 2}, \tilde{\mu}=\epsilon / n_{s} V$, and $z=Z / \xi$, one obtains the following for a real $f(z)$ :

$$
\frac{d^{2} f}{d z^{2}}=f^{3}-f
$$

if $z<0$, and

$$
\frac{d^{2} f}{d z^{2}}=f^{3}+\tilde{\mu} f
$$

if $z>0$. These equations can be readily solved using first integrals of motion with the boundary conditions $f(-\infty)=1$ and $f(\infty)=0$,

$$
\frac{d f}{d z}=-\left(\frac{1}{2}+\frac{f^{4}}{2}-f^{2}\right)^{1 / 2}
$$

and

$$
\frac{d f}{d z}=-\left(\tilde{\mu} f^{2}+\frac{f^{4}}{2}\right)^{1 / 2},
$$

for $z<0$ and $z>0$, respectively. The solution in the superconducting electrode is given by

$$
f(z)=\tanh \left[-2^{-1 / 2} z+0.5 \ln \frac{2^{1 / 2}(1+\tilde{\mu})^{1 / 2}+1}{2^{1 / 2}(1+\tilde{\mu})^{1 / 2}-1}\right] .
$$

It decays in the close vicinity of the barrier from 1 to $f(0)$ $=[2(1+\tilde{\mu})]^{-1 / 2}$ in the interval about the coherence length $\xi$. On the other side of the boundary, $z>0$, it is given by

$$
f(z)=\frac{(2 \tilde{\mu})^{1 / 2}}{\sinh \left(z \tilde{\mu}^{1 / 2}+\ln \left\{2[\tilde{\mu}(1+\tilde{\mu})]^{1 / 2}+[1+4 \tilde{\mu}(1+\tilde{\mu})]^{1 / 2}\right\}\right)} .
$$

Its profile is shown in Fig. 1. Remarkably, the order parameter penetrates the normal layer up to the length $Z^{*}$ $\approx(\tilde{\mu})^{-1 / 2} \xi$, which could be larger than $\xi$ by many orders of magnitude, if $\tilde{\mu}$ is small. It is indeed the case if the barrier layer is sufficiently doped. For example, taking $x^{\prime}=0.1$, $c$-axis $m_{c}=2000 m_{e}$, in-plane $m=10 m_{e},{ }^{11} a=0.4 \mathrm{~nm}$, and $\xi$ $=0.6 \mathrm{~nm}$ yields $T_{0} \approx 140 \mathrm{~K}$ and $(\tilde{\mu})^{-1 / 2} \gtrsim 50$ at $T=25 \mathrm{~K}$. Hence, the order parameter could penetrate the normal cuprate semiconductor up to a hundred coherence lengths or even more as observed. ${ }^{1}$ If the thickness of the barrier $L$ is small compared with $Z^{*}$ and $(\tilde{\mu})^{1 / 2} \ll 1$, the order parameter decays following the power law rather than exponentially,

$$
f(z)=\frac{\sqrt{2}}{z+2} .
$$

Hence, for $L \leqq Z^{*}$, the critical current should also decay following the power law as discussed below. On the other hand, for an undoped barrier, $\tilde{\mu}$ becomes larger than unity, $\tilde{\mu}$ $\propto \ln \left(m T / \pi x^{\prime}\right) \rightarrow \infty$ for any finite temperature $T$ when $x^{\prime} \rightarrow 0$, and the current should exponentially decay with the charac- 
teristic length smaller that $\xi$, as is experimentally observed as well. ${ }^{4}$

To get more insight in the temperature and barrierthickness dependence of the critical current, one has to solve the GP equation in the $S N^{\prime} S$ junction geometry (Fig. 2) with the current $J=\left(2 e n_{s} / m_{c} \xi\right) R^{2} d \Theta / d z$. Here, $R(z)$ and $\Theta(z)$ are the amplitude and the phase of the order parameter, respectively, and $f(z)=R(z) \exp (i \Theta(z))$. The current does not depend on $z$, so that the equation can be written as

$$
\frac{d^{2} R}{d z^{2}}=R^{3}-\left(1-\frac{j^{2}}{R^{4}}\right) R
$$

if $|z|>l$, and

$$
\frac{d^{2} R}{d z^{2}}=R^{3}+\left(\tilde{\mu}+j^{2} / R^{4}\right) R
$$

if $|z|<l$, where $l=L / \xi$ and $j=J m_{c} \xi / 2 e n_{s}$. Using integrals of motion with the boundary conditions $d R / d z=0$ at $z=0$ $[R(z)=R(-z) \quad$ since the junction is symmetric $], R_{\infty}^{3}$ $=R_{\infty}-j^{2} / R_{\infty}^{3}$, and $d R_{\infty} / d z=0$, these equations are reduced to

$$
\frac{d R}{d z}=\left[\frac{\left(R^{4}-R_{0}^{4}\right)}{2}+\tilde{\mu}\left(R^{2}-R_{0}^{2}\right)+j^{2}\left(R_{0}^{-2}-R^{-2}\right)\right]^{1 / 2},
$$

for $0<z<l$, and

$$
\frac{d R}{d z}=\left[\frac{\left(R^{4}-R_{\infty}^{4}\right)}{2}+\left(R_{\infty}^{2}-R^{2}\right)+j^{2}\left(R_{\infty}^{-2}-R^{-2}\right)\right]^{1 / 2},
$$

for $z>l$. Then, integrating Eq. (14) from $z=0$ up to $z=l$ allows us to connect the current and the order parameter $R_{0}$ in the center of the barrier at $z=0$,

$$
\int_{1}^{R_{l}^{2} / R_{0}^{2}} \frac{d x}{\sqrt{x\left(x^{2}-1\right)+x(x-1) \alpha+(x-1) \beta}}=\sqrt{2} R_{0} l .
$$

Since $R_{l} \gg R_{0}$ for sufficiently thick barrier, $l \gg 1$, the upper limit in this integral can be extended to $\infty$, yielding a transcendental equation for $R_{0}$ as a function of $j$ as follows:

$$
{ }_{2} F_{1}\left[\frac{1}{2}, \frac{1}{2} ; 1 ; \frac{1}{2}\left(1-\frac{3+\alpha}{2 \sqrt{\alpha+\beta+2}}\right)\right]=\sqrt{2}(\alpha+\beta+2)^{1 / 4} \frac{R_{0} l}{\pi}
$$

where ${ }_{2} F_{1}(a, b ; c ; z)$ is the Gauss hypergeometric function, $\alpha=2 \tilde{\mu} / R_{0}^{2}$ and $\beta=2 j^{2} / R_{0}^{6}$. When $\alpha=\beta=0$, one obtains $R_{0} \propto 1 / l$, as in Eq. (11), and when $\alpha \rightarrow \infty$, the order parameter decays exponentially as in Eq. (10).

To evaluate the critical current, one can reduce the integral in Eq. (16) to a single-parameter integral $I(p)$ $=\int_{0}^{\infty} d x\left[x(x+1)^{2}+p x\right]^{-1 / 2}$, with $p=-1+4(\alpha+\beta+2) /(3+\alpha)^{2}$. This integral can be analytically approximated (see Fig. 2) as $I(p) \approx 3.3(1.1+p)^{-1 / 4}$, if $p$ is not too close to -1 . Then, solving

$$
I(p)=R_{0} l(3+\alpha)^{1 / 2},
$$

with respect to the current, yields $j \approx R_{0}\left(25 / 2 l^{4}-R_{0}^{4}\right.$ $\left.-\tilde{\mu} R_{0}^{2}\right)^{1 / 2}$ and the critical current $j_{c} \approx 4\left(1-2 \tilde{\mu} l^{2}\right) / l^{3}$. These

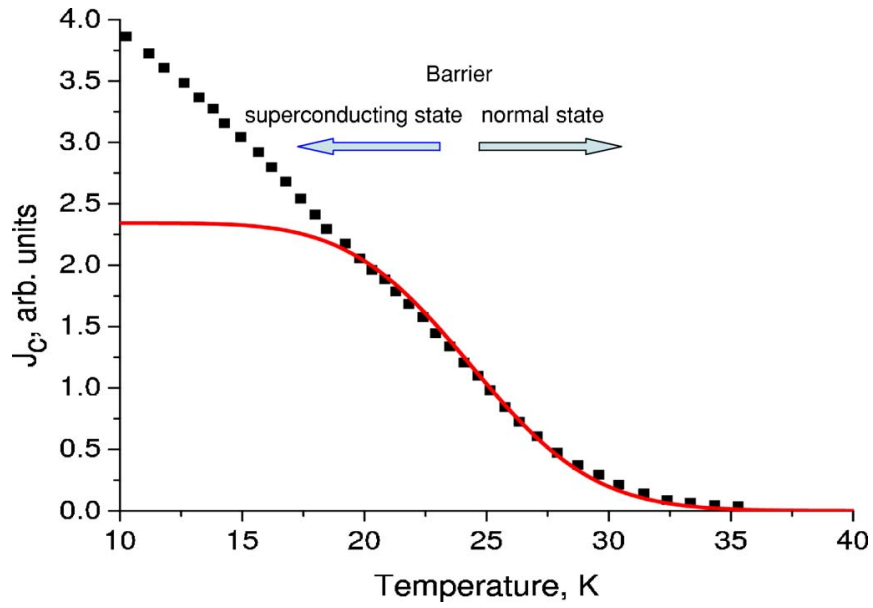

FIG. 3. (Color online) Temperature dependence of the critical current of the trilayer $S N^{\prime} S$ device (Ref. 1) with $L=5 \mathrm{~nm}$ (symbols) described by Eq. (19) at $T>T_{c}^{\prime}$.

expressions are applied when $l \leqq(\tilde{\mu})^{-1 / 2}$ (i.e., $L \leqq Z^{*}$ ).

To get the temperature dependence of the critical current $J_{c}(T)$ in the whole range of parameters, one can apply the scaling $j_{c} \propto R_{0}^{2} / l$, where $R_{0}$ is defined as the exact solution of the $S N^{\prime}$ boundary problem [Eq. (10)] at $z=l, R_{0}=f(l)$. In such a way one finds (in ordinary units)

$$
J_{c}(T)=\frac{A \epsilon(T)}{k_{B} \sinh ^{2}\left[L \sqrt{2 m_{c} \epsilon(T)} / \hbar\right]},
$$

where $A \approx 4 e k_{B} \xi_{0}^{2} n_{s 0} / \hbar L$ is temperature independent. With the zero-temperature coherence length $\xi_{0}=0.5 \mathrm{~nm}$ and condensate density $n_{s 0}=x / 2 \Omega$, one estimates $A$ $\approx 150 \mathrm{kA} / \mathrm{cm}^{2} \mathrm{~K}$ for optimum doping $x=0.15$ and the barrier thickness $2 L=10 \mathrm{~nm}$ (here, $\Omega$ is the unit cell volume). Equation (19) fits well the temperature dependence and absolute values of the critical current (Fig. 3), measured by Bozovic et al. ${ }^{1}$ with $T_{0}=160 \mathrm{~K}$ and $m_{c}=1000 m_{e}$ (Fig. 3). The typical device of Ref. 1 used $\mathrm{La}_{1.85} \mathrm{Sr}_{0.15} \mathrm{CuO}_{4}$ with $T_{c}$ $\approx 45 \mathrm{~K}$ as the superconducting electrodes, while the normal $N^{\prime}$ barrier was made of underdoped $\mathrm{La}_{2} \mathrm{CuO}_{4+\delta}$ with typical $T_{c}^{\prime} \approx 25 \mathrm{~K}$. One can see from Fig. 3 that the theory describes the critical current in the normal region of the barrier, where $\tilde{\mu}$ is positive. When the barrier becomes superconducting (approximately below $20 \mathrm{~K}$ ), the experimental critical current naturally exceeds the theoretical "semiconducting" $J_{c}(T)$.

A possibility of real-space pairing, originally proposed by $\mathrm{Ogg}^{20}$ and later by Schafroth and Blatt and Butler, ${ }^{21}$ has been the subject of many discussions as opposed to the Cooper pairing, particularly heated over the last 20 years after the discovery of high-temperature superconductivity in cuprates. Our extension of the BCS theory toward the strong interaction between electrons and ion vibrations proved that BCS and Ogg-Schafroth pictures are two extreme limits of the same problem. For a very strong electron-phonon coupling, polarons become self-trapped on a single lattice site and bipolarons are on-site singlets. In the Holstein model of the electron-phonon interaction, their mass appears only in the 
second order of polaron hopping, so that on-site bipolarons are very heavy. This estimate led some authors to the conclusion that the formation of itinerant small polarons and bipolarons in real materials is unlikely, ${ }^{22}$ and hightemperature bipolaronic superconductivity is impossible. ${ }^{23}$ However, we have noticed that the Holstein model is an extreme polaron model and typically yields the highest possible value of the (bi)polaron mass in the strong coupling regime. Cuprates are characterized by poor screening of highfrequency optical phonons and are more appropriately described by the long-range Fröhlich electron-phonon interaction. ${ }^{5}$ The unscreened Fröhlich electron-phonon interaction provides relatively light small polarons and bipolarons, which are several orders of magnitude lighter than small Holstein (bi)polarons.

I conclude that the bipolaron theory accounts for GPE and NPE in slightly doped semiconducting and undoped insulating cuprates, respectively. It predicts the occurrence of a length scale $\hbar / \sqrt{2 m_{c} \epsilon(T)}$. In a wide temperature range far from the transition point, $T_{c}^{\prime}<T<T_{0}$, this length turns out to be much larger than the zero-temperature coherence length if bosons are almost 2D. The physical reason why the quasi-2D bosons display a large normal-state coherence length, whereas three-dimensional (3D) Bose systems (or
$N$-dimensional Fermi systems) at the same values of parameters do not, originates in the large DOS near the band edge of bosons compared with 3D DOS. Since DOS is large, the chemical potential is pinned near the edge with the magnitude $\epsilon(T)$, which is exponentially small when $T<T_{0}$. Importantly, the theory predicts an unusual dependence of $J_{c}$ on the barrier thickness $2 L$; in particular, at low temperatures, $T<T_{c}^{\prime}$, when the barrier is superconducting, there is almost no $L$ dependence, above the transition of the barrier, $J_{c}$ $\propto 1 / L^{3}$, and well above the transition $\left(T \gtrsim T_{0} \gg T_{c}^{\prime}\right), J_{c}$ decays exponentially with $L$. Based on a great number of experimental observations ${ }^{11}$ including GPE and NPE, I argue that the most likely scenario for superconducting cuprates is the genuine Bose-Einstein condensation of real-space mobile lattice bipolarons.

I thank A. F. Andreev, I. Bozovic, L.P. Gor'kov, and V. V. Kabanov for valuable discussions and E. V. Ferapontov, S. Flach, V. Khodel, F. V. Kusmartsev, and A. P. Veselov for illuminating comments on integrability conditions for differential systems. The work was supported by EPSRC (UK) (Grants No. EP/C518365/1, No. EP/D035589/1, and No. EP/ D07777X/1).
${ }^{1}$ I. Bozovic, G. Logvenov, M. A. J. Verhoeven, P. Caputo, E. Goldobin, and M. R. Beasley, Phys. Rev. Lett. 93, 157002 (2004), and references therein.

${ }^{2}$ P. Abbamonte, L. Venema, A. Rusydi, G. A. Sawatsky, G. Logvenov, and I. Bozovic, Science 297, 581 (2002).

${ }^{3}$ The BCS theory can lead to a large proximity effect only if the normal layer is very close to its superconducting transition [J. Quintanilla, K. Capelle, and L. N. Oliveira, Phys. Rev. Lett. 90, 089703 (2003)].

${ }^{4}$ I. Bozovic, G. Logvenov, M A. J. Verhoeven, P. Caputo, E. Goldobin, and T. H. Geballe, Nature (London) 422, 873 (2003).

${ }^{5}$ A. S. Alexandrov, Phys. Rev. B 53, 2863 (1996).

${ }^{6}$ D. Mihailovic, C. M. Foster, K. Voss, and H. J. Heeger, Phys. Rev. B 42, 7989 (1990).

${ }^{7}$ P. Calvani, M. Capizzi, S. Lupi, P. Maselli, A. Paolone, P. Roy, S. W. Cheong, W. Sadowski, and E. Walker, Solid State Commun. 91, 113 (1994).

${ }^{8}$ R. Zamboni, G. Ruani, A. J. Pal, and C. Taliani, Solid State Commun. 70, 813 (1989).

${ }^{9}$ G. M. Zhao and D. E. Morris, Phys. Rev. B 51, 16487 (1995); G.-M. Zhao, M. B. Hunt, H. Keller, and K. A. Müller, Nature (London) 385, 236 (1997); R. Khasanov, D. G. Eshchenko, H. Luetkens, E. Morenzoni, T. Prokscha, A. Suter, N. Garifianov, M. Mali, J. Roos, K. Conder, and H. Keller, Phys. Rev. Lett. 92, 057602 (2004).

${ }^{10}$ A. Lanzara, P. V. Bogdanov, X. J. Zhou, S. A. Kellar, D. L. Feng, E. D. Lu, T. Yoshida, H. Eisaki, A. Fujimori, K. Kishio, J. I.
Shimoyana, T. Noda, S. Uchida, Z. Hussain, and Z. X. Shen, Nature (London) 412, 510 (2001); G-H. Gweon, T. Sasagawa, S. Y. Zhou, J. Craf, H. Takagi, D.-H. Lee, and A. Lanzara, ibid. 430, 187 (2004); X. J. Zhou et al., Phys. Rev. Lett. 95, 117001 (2005).

${ }^{11}$ A. S. Alexandrov, Theory of Superconductivity: From Weak to Strong Coupling (IOP, Bristol, 2003).

${ }^{12}$ A. S. Alexandrov, Int. J. Mod. Phys. B 14, 3298 (2000).

${ }^{13}$ A. F. Andreev, Pis'ma Zh. Eksp. Teor. Fiz. 79, 100 (2004).

${ }^{14}$ A. S. Alexandrov and V. N. Zavaritsky, Phys. Rev. Lett. 93, 217002 (2004); A. S. Alexandrov, ibid. 95, 259704 (2005).

${ }^{15}$ A. S. Alexandrov, Phys. Rev. Lett. 96, 147003 (2006).

${ }^{16}$ A. S. Alexandrov, Phys. Rev. B 60, 14573 (1999).

${ }^{17}$ E. P. Gross, Nuovo Cimento 20, 454 (1961); L. P. Pitaevskii, Zh. Eksp. Teor. Fiz. 40, 646 (1961) [Sov. Phys. JETP 13, 451 (1961)].

${ }^{18}$ M. Yu. Kagan and D. V. Efremov, Phys. Rev. B 65, 195103 (2002).

${ }^{19}$ V. N. Popov, Theor. Math. Phys. 11, 565 (1972); D. S. Fisher and P. C. Hohenberg, Phys. Rev. B 37, 4936 (1988).

${ }^{20}$ R. A. Ogg, Jr., Phys. Rev. 69, 243 (1946).

${ }^{21}$ M. R. Schafroth, Phys. Rev. 100, 463 (1955); J. M. Blatt and S. T. Butler, ibid. 100, 476 (1955).

${ }^{22}$ E. V. L. de Mello and J. Ranninger, Phys. Rev. B 58, 9098 (1998).

${ }^{23} \mathrm{P}$. W. Anderson, The Theory of Superconductivity in the Cuprates (Princeton University Press, Princeton, NJ, 1997). 